\title{
Automatic Stabilization of an Adaptive Feedback Control for Noise Cancelling In-ear Headphones
}

\author{
Sven Höber, Christian Pape and Eduard Reithmeier \\ Institute of Measurement and Automatic Control, Leibniz University Hanover, Hanover 30167, Germany
}

\begin{abstract}
Headphones with an integrated active noise cancellation system have been increasingly introduced to the consumer market in recent years. When exposing the human ear to active noise sources in this striking distance, the ensuring of a safe sound pressure level is vital. In feedback systems, this is coupled with the stability of the closed control loop; stable controller design is thus essential. However, changes in the control path during run-time can cause the stable control system to become unstable, resulting in an overdrive of the speakers in the headphones. This paper proposes a method, which enables the real-time analysis of the current system state and if necessary stabilizes the closed loop while maintaining the active noise reduction. This is achieved by estimating and evaluating the open loop behavior with an adaptive filter and subsequently limiting the controller gain in respect to the stability margin.
\end{abstract}

Key words: Active noise cancellation, feedback control, stability analysis.

\section{Introduction}

Especially when at work or travelling long distances, by plane for example, ambient noise quickly becomes a severe source of stress [1]. Here, the use of hearing protection or headphones with ANC (active noise cancellation) derives a substantial gain in comfort. While passive control approaches can only provide reduction of high frequency sounds, ANC is capable of additionally reducing the low frequency parts [2] — such as exposed by engines or industrial machines in factories. Hence, this technology has been an issue of interest for over 80 years [3]. Nowadays it is already used in pilot headsets for example, but it is also offered in commercially available headphones to provide silence in every desirable situation.

For the technical implementation of ANC headphones based on a feedback control structure, the interference signal of ambient noise and speaker sound is measured by an internal microphone and fed back to an integrated controller. However, a stable feedback system can become unstable during run-time, which in this application is expressed in a sudden overdrive and

Corresponding author: Sven Höber, M.Sc., research fields: active noise control, and acoustic systems identification. eventual destruction of the speakers or even damage of the user's inner ear. Here, this problem is caused by a change in the acoustical transfer path between the speaker and the microphone, induced even by slight variations of the headphones' positions in the ear. In Ref. [4], this problem is overcome by limiting the controller gain in respect to the speaker's driving signal power. Additionally, practical applications in general limit the actual speaker output or at least deactivate the controller in case of an imminent overdrive. Thus, conventional solutions either act preemptively or in response to the already unstable state, still exposing the listener to a potential danger. Hence, there is the need to continuously observe the behavior of the control loop during run-time in order to detect the possibly occurring unstable state at an early stage.

This paper presents the successive composition of a method that automatically stabilizes an unstable feedback control for ANC in-ear headphones, eventually protecting the listener from any harm caused by the typically overdriving speakers in that case. Additionally, the stabilized system is still capable of reducing ambient noise since the controller gain is hold on a lower but non-zero level. To track the changes in the secondary path, which cause the closed loop to become unstable, at 
first a respective online estimation is presented in Section 3.2. In turn, this model is used to derive an estimate of the open loop transfer function that is then evaluated to check the stability of the system (Section 3.3). Finally, Section 3.4 proposes the application of an adaptive maximum controller gain, used to retrieve the stability of the system after it has become unstable. The following sections 4 and 5 present the results of the components applied in simulations and to the real ANC in-ear headphones system itself.

\section{System Setup}

The investigated system is a feedback control for active noise cancelling in-ear headphones. A schematic of the principal setup and an equivalent block diagram are presented in Fig. 1. The aim is to attenuate the ambient noise $d(k)$ around the internal error microphone located inside the silicone nozzle of the headphones. For this, an inversely phased sound, the so called anti-noise $u(k)$, is emitted by the regular speaker to cause a destructive interference of anti-noise and ambient noise. The speaker is driven with the actuating variable $y(k)$, which is continuously calculated by the controller.

Basically, the optimal controller for this task is derived from the minimization of the error signal power $\mathrm{E}\{e(k)\}$ for a certain given noise $d(k)$. If the noise spectrum changes, the controller is adapted again to ensure optimal attenuation. However, the actuating variable (put out by the controller) first has to pass the control path $S(z)$ - in the field of ANC referred to as secondary path-before it arrives at the error microphone as the anti-noise. Since the sound is distorted during its propagation through the secondary path, the transfer behavior $S(z)$ must also be incorporated into the controller design. Now, if the secondary path changes, the originally designed control loop is not assured to be stable anymore. As mentioned in Section 1, this can happen anytime due to a varied headphone position in the ear.

To at least keep track of the magnitude variation in
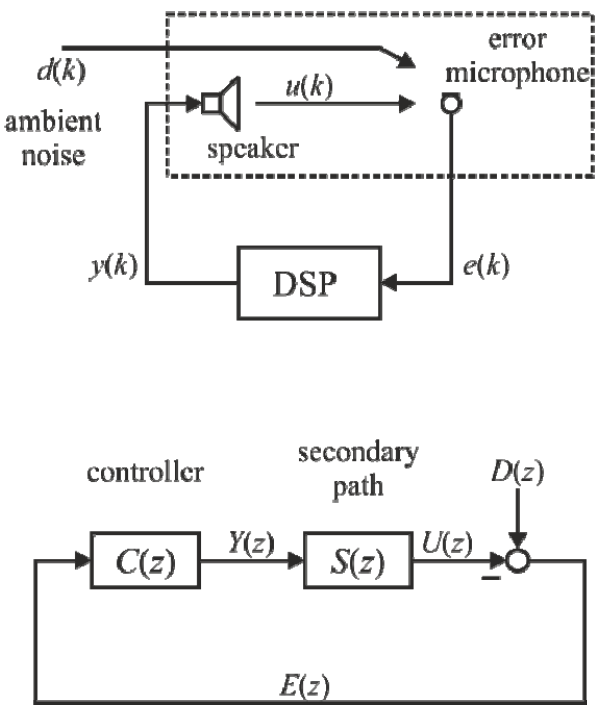

Fig. 1 Top: schematic of the ANC in-ear headphones system. Bottom: equivalent block diagram.

the secondary path, additionally the gain $K$ of the controller is adapted in respect to the actuating variable power $P_{y}(k)$ (see Ref. [4]), hence $K=K\left(P_{y}(k)\right.$ ). If the power crosses a critically high level, the gain is decreased or set to zero; in turn, if the power sinks below a certain minimum level, the gain is increased again and - if possible — set to its maximum value to retrieve the optimal performance. This way, the actuating variable is always held within a safe range regarding the corresponding speaker output.

Fig. 2 shows the hardware later used for the experimental investigation of the system (presented in Section 5). Instead of actual probands, the ANC headphones are applied to a HATS (Head and Torso Simulator), which recreates the transfer behavior of the human correspondent. This way, no test person is exposed to a possibly unstable active noise control system.

The actual feedback controller is implemented on a DSP (digital signal processor), contained in a real-time hardware that features several input and output channels, an LED front panel for optical user feedback and push buttons for the manual system control.

The experimental results obtained with this setup are presented in Section 5. First of all, the following section 

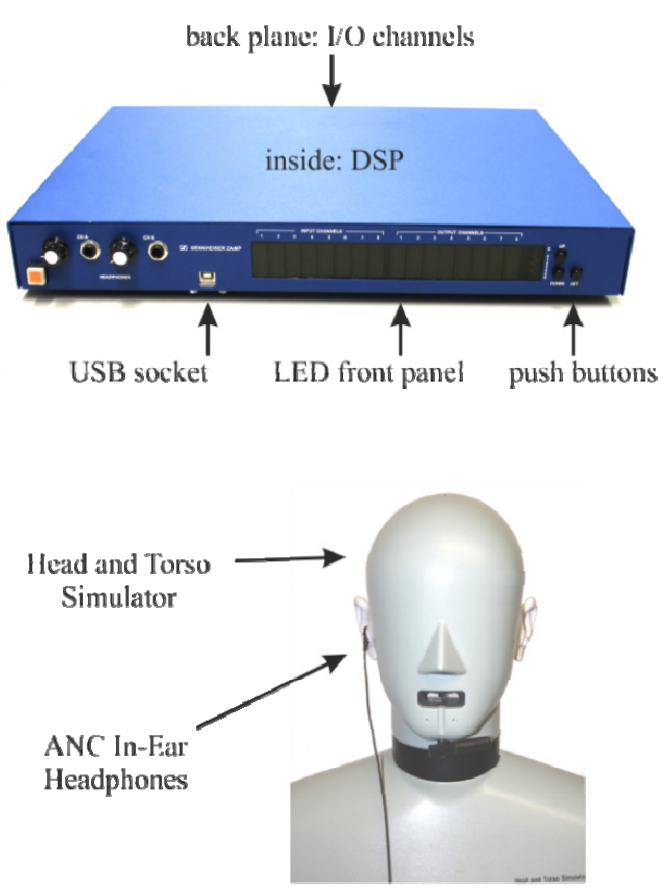

Fig. 2 Top: real-time hardware. Bottom: Head and Torso Simulator.

focuses on the theoretical solution of the mentioned problems, which is then tested in simulations (Section 4) before applied to the real system.

\section{Design of the Stabilization}

\subsection{Basic Considerations}

To specifically influence the behavior of the control system, it first has to be analyzed. For this, the signals $e(k)$ and $u(k)$ are needed to subsequently estimate the open loop transfer function

$$
G_{\mathrm{o}}(z)=S(z) C(z)=\frac{U(z)}{E(z)}
$$

The closed loop behavior-primarily its stability - can then be derived from the roots of the characteristic polynomial

$$
G_{\mathrm{o}}(z)+1=0
$$

which is eventually determined by the open loop. However, the signal $u(k)$ needed to calculate the transfer function (Eq. (1)) is not measurable particularly. Furthermore, a change of the secondary path behavior, contained in the open loop, makes an a priori measurement of its transfer function $S(z)$ before run-time obsolete. It is thus necessary to identify a model of the secondary path online (at run-time). Finally, a continuous estimation of the signal $u(k)$ can be obtained by filtering the actuating variable according to

$$
\hat{u}(k)=\hat{s}(k) * y(k)
$$

with $\hat{s}(k)$ being the impulse response of the estimated secondary path. The apparently easier way to calculate the open loop transfer function from the product $S(z) \cdot C(z)$ however involves a higher computational effort than the proposed convolution.

\subsection{Online Secondary Path Estimation}

The secondary path is parametrically modeled using an adaptive FIR filter. This ensures that the roots of the model filter are always inside the unit circle and no stability problems have to be taken into account at this point. Furthermore, the conventional LMS algorithm - known for its low effort in computation and implementation [5] — can be used for the adaptation of the filter parameters. The block diagram in Fig. 3 illustrates the parametrical identification of an LTI (linear time invariant) system using the LMS algorithm.

The aim of the algorithm is to adapt the filter such that it replicates the real system's behavior. For this, both are excited by an input signal $x(k)$-ideally featuring a constant spectral power density like white noise [6]. Then the filter output is continuously compared to the desired signal (the output of the identified system). The LMS algorithm now has the

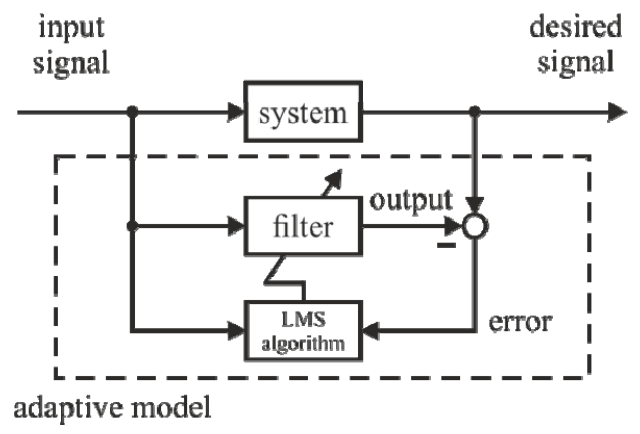

Fig. 3 General setup of system identification using the LMS algorithm. 
task to minimize the resulting error $e(k)$ by successively updating the filter coefficients $h_{i}$ according to

$$
\mathbf{h}(k+1)=\mathbf{h}(k)-\mu e(k) \mathbf{x}^{*}(k) .
$$

Applied to the investigated system (Fig. 4), the desired signal corresponds to the needed output $u(k)$, which is not available as mentioned in the previous section. Instead, the signal of the error microphone $e(k)$ is used as the desired signal. It still contains the information of the signal $u(k)$, but is disturbed by the noise $d(k)$, which the control is meant to reject. The influence of the disturbance on the convergence process of the LMS algorithm is picked up again in Section 3.3.

\subsection{Open Loop Estimation and Stability Analysis}

In order to analyze the control system regarding its stability it is necessary to obtain the open loop transfer function (see Eqs. (1) and (2)). However, the calculation using the z-transformed signals $\hat{U}(z)$ and $E(z)$ requires a high computational effort because of the mandatory FFT. Hence, the open loop is as well as the secondary path identified by means of an adaptive filter. This procedure is depicted in Fig. 5.

Again, an FIR filter is used to prevent stability problems during the adaption process. Furthermore, this way the model filter directly yields the coefficients $a_{m}$ of the characteristic polynomial

$$
\hat{G}_{\mathrm{o}}(z)+1=\sum_{m=0}^{M} a_{m} \cdot z^{m}+1
$$

Its coefficients can now be evaluated to check the stability of the model filter and the control loop respectively. This can be done by applying the determinant criterion of Jury, a conventional method for stability analysis of discrete time LTI systems - in dependence on the Hurwitz criterion for continuous time systems [7]. The aim is to constantly analyze the identified open loop using the Jury criterion to detect changes of the closed loop state during run-time. As soon as the changes result in a loss of the stability, action can be taken immediately this way.

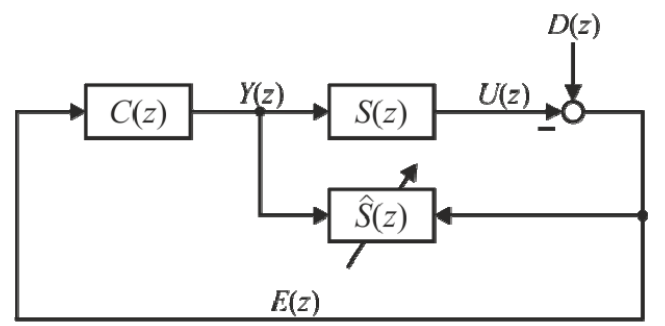

Fig. 4 Online secondary path estimation applied to the feedback control system.

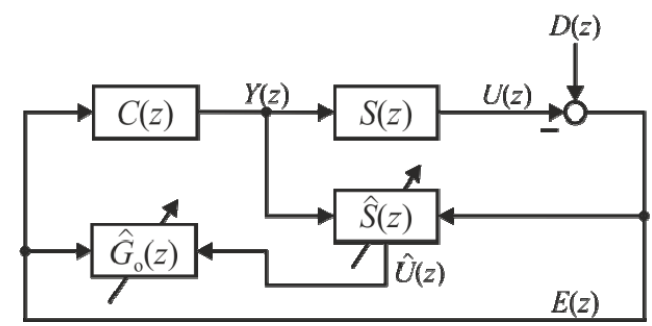

Fig. 5 Block diagram of the real-time open loop estimation.

However, there are two essential constraints that impair the application of the proposed stability analysis. First, the Jury criterion has a total computation effort of $2 M-3$ major operations (calculating the determinants) plus the subsequent evaluation of the stability test itself (the examination of possibly $M+1$ conditions). This effort can be reduced by an appropriate implementation, but the algorithm still requires considerable time for the intended filter lengths $(M>40)$ in respect to the available time between consecutive sample steps. Hence, a continuous analysis of the open loop coefficients is not assured.

The other constraint concerns the identification of the open loop and the coefficients $a_{m}$ respectively. Their validity strongly depends on the convergence of the adaption process, which is determined by the parameters of the LMS algorithm and the influence of the noise $d(k)$ as mentioned in Section 3.2. In other words, the result of the Jury stability test is only as reliable as the model filter represents the real system behavior. If the LMS algorithm does not converge, the stability test will yield no actual information about the system state.

The problems mentioned were specifically dealt with in Ref. [8], where a solution was proposed that 
enabled the real-time analysis of a feedback control by observing the coefficients of the estimated open loop directly without complex processing. In detail, it was shown that the distribution of the model filter coefficients characteristically depends on the state of the corresponding system. Analytically, the sign changes of the coefficients increase immediately when the identified system becomes unstable (this is illustrated later in Section 4). Regarding the application, we define a positive value $\xi$, which reflects the critical amount of coefficient sign changes $\psi(k)$ corresponding to the unstable state of the closed loop. By stating the assignment

$$
\text { state }=\left\{\begin{array}{cc}
\text { stable, } & \psi(k)<\xi \\
\text { unstable }, & \psi(k) \geq \xi
\end{array}\right.
$$

It is now possible to detect a state change without special processing of the coefficient values. Finally, the loop has to be stabilized again, which the following section is going to focus on.

\subsection{Reset of the Stability}

The easiest way to stabilize the control loop is to reduce its controller gain. However, without knowledge of the root locus it is not possible to both retrieve stability and sustain the still achievable performance. Moreover, the need of immediate action after detecting the unstable state makes a temporary zero gain inevitable. Thus, if the system analysis detects instability (see Section 3.3), the adaptive gain $K\left(P_{y}\right)$ is set to zero at first. Since the actuating variable power then decreases as well, the gain is subsequently increased again until it retrieves its original value (see Section 2). However, this case sets the system back to the unstable state, resulting in another temporary gain of zero. Finally, this repetitive behavior makes the system inoperative.

The seemingly obvious idea to simply re-adapt the controller filter in case of instability however fails because of the computational effort associated: first, the coefficients had to be updated by the adaption algorithm, which would take several operations leaving the system uncontrolled at this time. Second, once the new controller coefficients were calculated, the closed loop would necessarily have to be analyzed again regarding its stability - resulting in further latency.

Hence, a solution must be found that enables to influence the closed loop behavior while avoiding complex computations. One way is to use the gain $K\left(P_{y}\right)$ but make its increase stop before the system gets unstable again. This can be achieved by additionally adapting the maximum value of the gain $K_{\max }$ also depending on the power of the actuating variable $P_{y}(k)$ (Fig. 6).

The aim is to limit the controller gain lest it results in the crossing of the critical power threshold reflecting a change to the unstable state. For this, the gain is raised until the power $P_{y}(k)$ reaches a value close to but lower than the critical threshold: when the system gets unstable (time a)) the gain is immediately set to zero and then increased as usual at first (period b)). As soon as the power hits the lower threshold $P_{\mathrm{LT}}$, the gain is hold on its current value, additionally reduced by a $10 \%$ safety margin - this is the new maximum gain level

$$
\tilde{K}_{\max }=0.9 \cdot K\left(P_{y}=P_{\mathrm{LT}}\right) .
$$

In the following (period c)), the gain is as usual controlled by the current value $P_{y}(k)$ again, but is now limited to its new maximum $\tilde{K}_{\max }$.

\section{Simulation Results}

Now, all components of the automatic stabilization (Sections 3.2 to 3.4 ) are tested in simulations. To
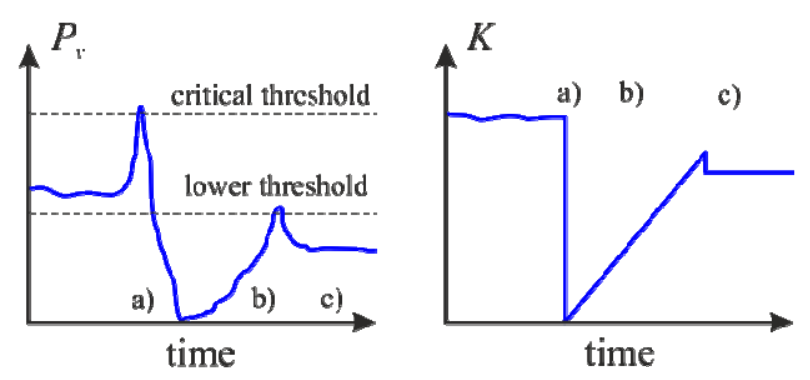

Fig. 6 Principal of the proposed adaptive maximum controller gain. 


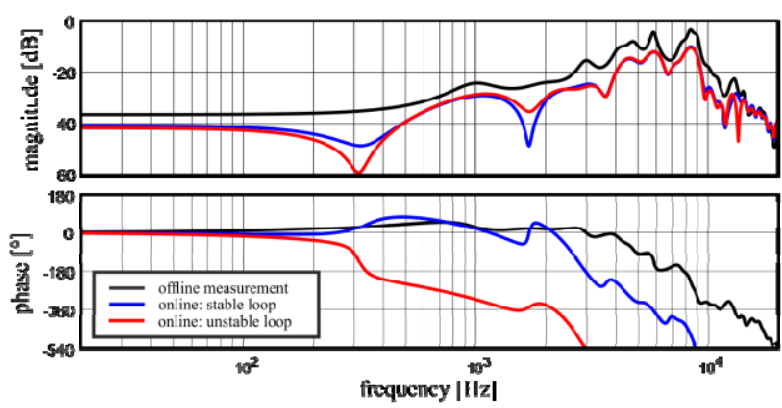

Fig. 7 Simulation result of the secondary path estimation.

induce the unstable case of the control, a delay is added to the secondary path after half the simulation time. First of all, Fig. 7 shows the results of the identification of the secondary path with the LMS algorithm before and after the closed loop becomes unstable.

However, before the presented results were obtained, it had shown that the level of the noise $d(k)$ and the spectral properties of the excitation signal-in the closed loop, the actuating variable $y(k)$-have a significant influence on the adaption process. Hence, the LMS algorithm for the online identification was initialized with an offline measurement of the secondary path

$$
\left.\mathbf{h}(k=0)\right|_{\text {online }}=\left.\mathbf{h}\right|_{\text {offline }}
$$

to force the convergence. The result shown in the Bode diagram above (Fig. 7) was obtained using this offline initialized adaption. Except for a slight constant difference in magnitude and a phase error for frequencies beyond $1 \mathrm{kHz}$, the behavior of the secondary path is modeled sufficiently. Especially the additional delay, which leads to an unstable control loop, is apparent in the phase diagram.

Subsequently, the open loop is identified. Fig. 8 shows the Bode diagram of the respective adapted filter. As for the secondary path, the LMS algorithm was used. The reference (black curves) is given by the frequency response of the calculated open loop transfer function regarding Eq. (1). Except for the magnitude of the stable case model (blue curves), the frequency responses of the identified filters do not approximate the behavior of the open loop sufficiently; the unstable

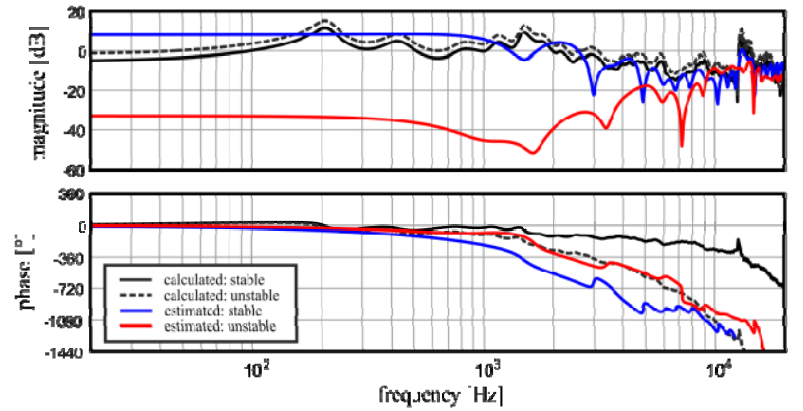

Fig. 8 Simulation result of the open loop estimation.

case model (red curves) shows a constant magnitude error up to $60 \mathrm{~dB}$ in the area of 1 to $2 \mathrm{kHz}$.

Compared to the calculated open loop, the model filter does not yield an adequate estimate of the system behavior. Yet, it represents the change in the state of the system, which also becomes apparent from the coefficients distribution, as discussed in Section 3.3 [8]. This is illustrated in Fig. 9 (since all depicted signals are numeric values directly read from the DSP, they lack physical units). It can be seen, how the destabilization affects the adaption of the model filter. The sign changes of the coefficient values increase as soon as the closed loop becomes unstable. This way (using Eq. (6)), the system state can be estimated at run-time after all.
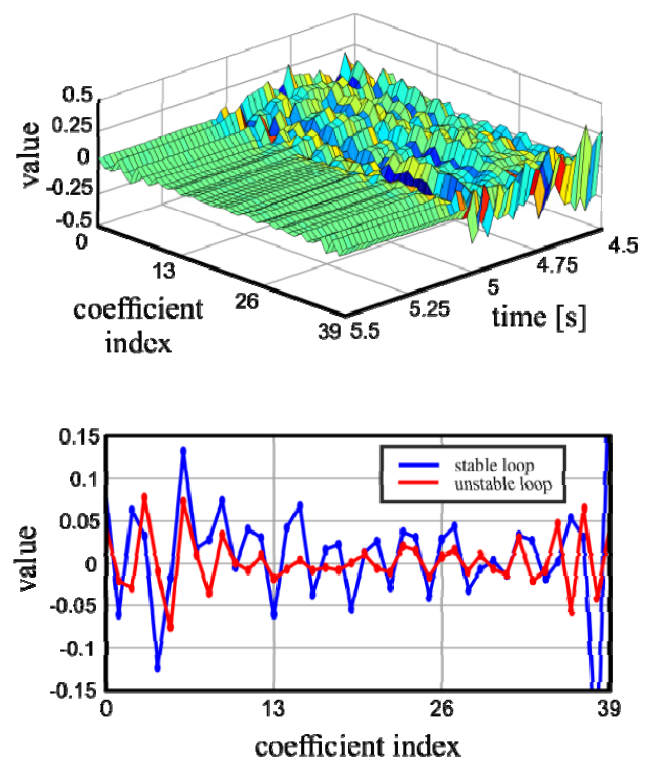

Fig. 9 Top: coefficients distribution of the open loop model filter (linearly interpolated) over time. Bottom: distribution at stable state compared to unstable state. 

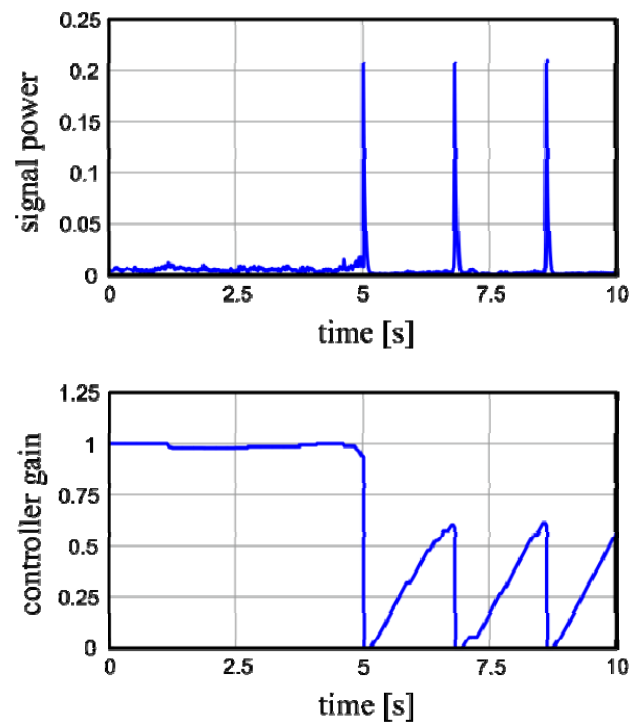

Fig. 10 Behavior of the controller gain with unstable closed loop.

Finally, the proposed adaptive maximum gain is simulated. To motivate the need for this action once again, Fig. 10 shows the effect of the state change on the adaptive controller gain $K$ without limiting its maximum level.

As soon as the closed loop becomes unstable after 5 $\mathrm{s}$, the estimated signal power of the actuating variable $P_{y}(k)$ (top) increases rapidly, forcing the gain $K$ (bottom) to be set to zero. Subsequently, when the loop is temporarily stable, the gain rises as intended, however crossing the critical threshold again which results in an unstable loop. If not interrupted by turning off the system, this procedure repeats itself endlessly.

Now, the adaptive maximum gain limits the value which the gain is set back to after a change of the state (Fig. 11). While the system is stable, the gain is adapted in respect to the current signal power $P_{y}(k)$ as usual; the maximum $K$-level is always retrieved. Now, when the system is destabilized $(t>5 \mathrm{~s})$, the gain is immediately set to zero and the adaption phase of the new maximum level begins. As soon as the power of the actuating variable $P_{y}(k)$ hits the lower threshold (here, $P_{\mathrm{LT}}=$ $0.005)$, the new value for $K_{\max }$ is set according to Eq. (7).

As shown on the right, it is about $\tilde{K}_{\max }=0.5$, which corresponds to $50 \%$ of the original maximum gain (additionally noticeable from the reduced actuating
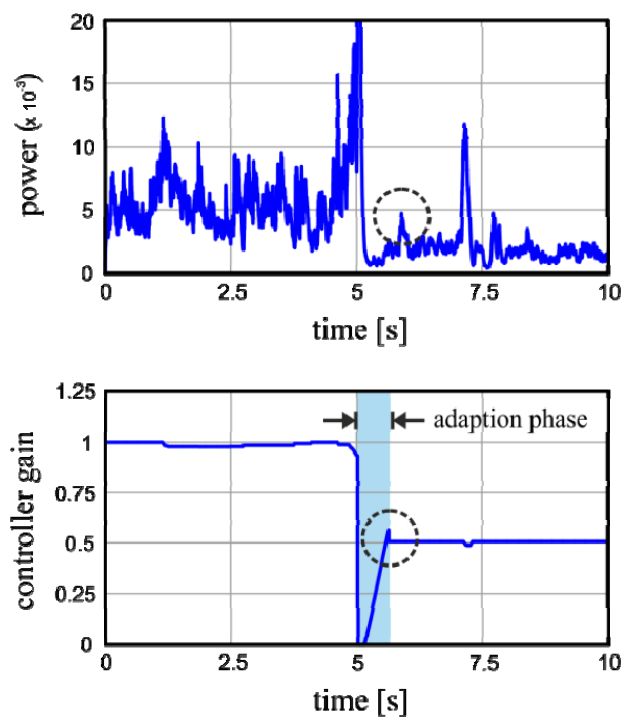

Fig. 11 Behavior of the controller gain when applying the adaptive maximum gain.

variable power level). Hence, the performance is in fact decreased, but for the benefit of a constantly stable control loop without having to stop the active noise reduction totally.

\section{Experimental Results}

Finally, the proposed methods were implemented on the DSP (digital signal processor) of the real-time hardware. The following Figs. 12 and 13 show the results of the online secondary path estimation and the subsequent open loop estimation obtained with the real ANC headphones system. Similarly to the simulation results, the secondary path (top) is approximated sufficiently, whereas the frequency response of the open loop (bottom) does not really match the calculated one.

However, the coefficients of the open loop model filter (Fig. 13, bottom) reflect the system state as observed in the simulation (see Fig. 9), while the secondary path model (top) is not affected by the loss of the stability. Hence, the real-time analysis of the ANC system can be performed as proposed (see Eq. (6))

At last, the closed loop is to be stabilized by applying the adaptive maximum gain. Fig. 14 shows, how the actuating variable power and the adaptive gain respectively act in case of a state change of the closed 

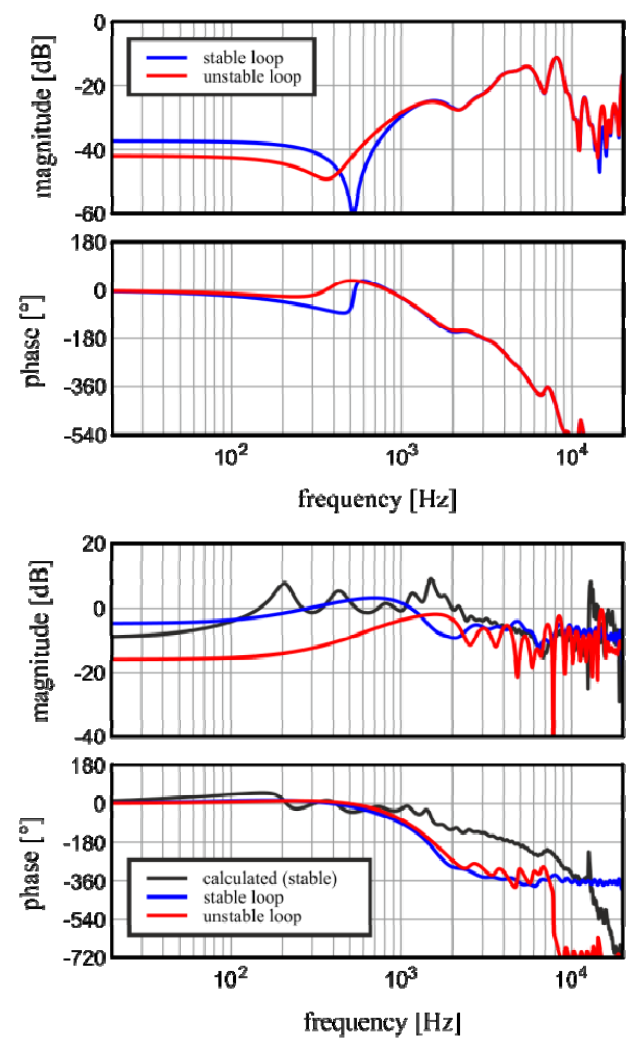

Fig. 12 Top: bode diagram of the identified real secondary path. Bottom: bode diagram of the respective estimated open loop.
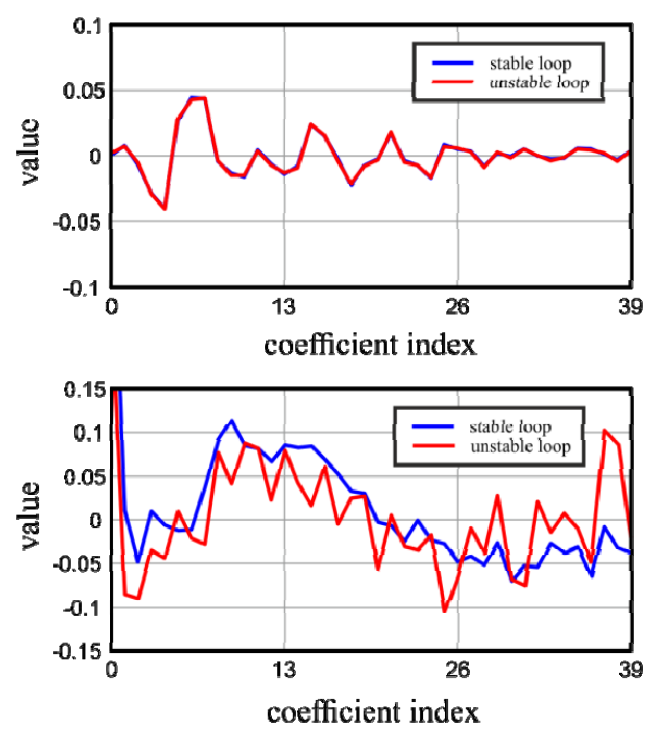

Fig. 13 Top: coefficients of the secondary path model. Bottom: coefficients of the open loop model.

loop. Basically, the behavior of the controller in the real system is again identical to the result obtained in the simulation (see Fig. 11). After the closed loop is
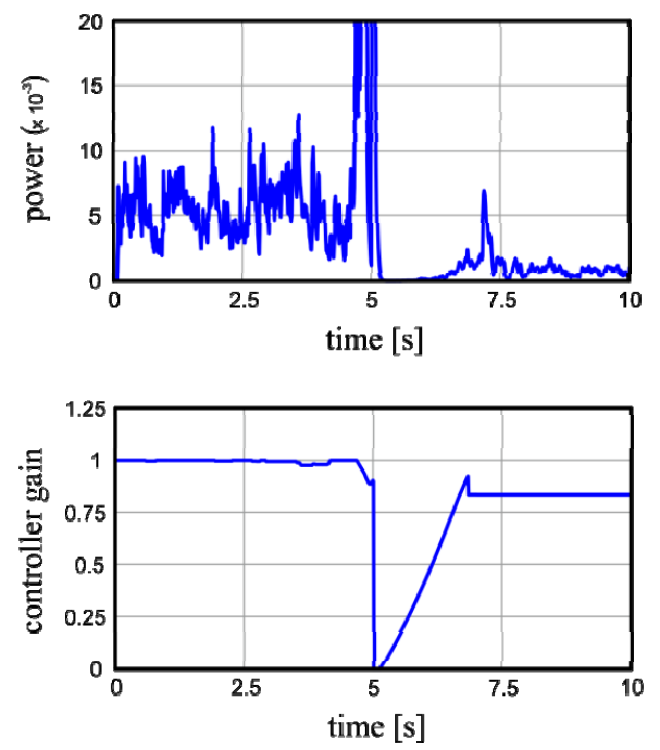

Fig. 14 Stabilization of the real control system with applied adaptive maximum gain..

destabilized, the adaption of the maximum value $K_{\max }$ begins. In a different way from the simulation result, the value reached now is bigger $\left(\tilde{K}_{\max } \approx 0.8\right.$ ), yet the power level of the actuating variable is lower. This results from the different way the real system is destabilized; since the manual variation of the headphones' positions in the HATS really induces a distortion of the magnitude in addition to the delay, the proportion between $\mathrm{K}$ and $\mathrm{y}$ also varies. However, the closed loop has been stabilized constantly and thus the adaptive maximum gain has proven to be effective.

\section{Summary}

Topic of this paper was the design of a method that enables the automatic and permanent stabilization of a feedback control system for active noise cancellation in-ear headphones. The first problem was to track changes of the secondary path (the control plant) behavior due to variations of the headphones' positions in the ears. For this, the path is identified online as an adaptive FIR filter using the LMS algorithm. To eventually derive the influence on the closed loop, the open loop behavior has to be observed. Similarly to the identification of the secondary path, the open loop transfer function is also estimated by means of an LMS 
adapted FIR filter. However, it was shown that the open loop model does not approximate the calculated frequency response sufficiently. Yet, it was possible to derive the current system state from the coefficients distribution of this model filter. The change in the closed loop behavior from stable to unstable could thus be detected at run-time after all. Eventually, when the system became unstable, it was able to be stabilized again by means of an adaptive maximum controller gain, which was limited to a new value close below but in a safe distance to the stability margin. This way, the control system was automatically stabilized while maintaining its functionality regarding noise reduction.

\section{References}

[1] Fastl, H., and Zwicker, E. 2007. Psychoacoustics-Facts and Models. Berlin and Heidelberg, Germany: Springer-Verlag.

[2] Kuo, S. M., and Morgan, D. R. 1999. "Active Noise Control-A Tutorial Review." In Proceedings of the IEEE 87: 943-73.

[3] Snyder, S. D. 2000. Active Noise Control Primer. New York, NY, USA: Springer Science + Business Media.

[4] Bruhnken, C. 2013. Adaptive Feedback-Regelung zur aktiven Lärmreduktion mit In-Ear-Kopfhörern. Garbsen, Germany: PZH-Verlag.

[5] Kuo, S. M., and Morgan, D. R. 1996. Active Noise Control Systems: Algorithms and DSP Implementations. New York, NY, USA: John Wiley \& Sons, Inc.

[6] Kammeyer, K. D., and Kroschel, K. 2012. Digitale Signalverarbeitung. Wiesbaden, Germany: Springer Vieweg.

[7] Lutz, H., and Wendt, W. 2012. Taschenbuch der Regelungstechnik. Frankfurt am Main, Germany: Verlag Harri Deutsch.

[8] Höber, S. 2015. "Real-Time Detection of Unstable Control Loop Behavior.” Engineering 7: 796-802. 